\title{
Optimized x-ray source scanning trajectories for iterative reconstruction in high cone-angle tomography
}

Andrew M. Kingston, Glenn R. Myers, Shane J. Latham, Heyang Li, Jan P. Veldkamp, et al.

Andrew M. Kingston, Glenn R. Myers, Shane J. Latham, Heyang Li, Jan P. Veldkamp, Adrian P. Sheppard, "Optimized x-ray source scanning trajectories for iterative reconstruction in high cone-angle tomography," Proc. SPIE 9967, Developments in X-Ray Tomography X, 996712 (4 October 2016); doi: $10.1117 / 12.2238297$

SPIE Event: SPIE Optical Engineering + Applications, 2016, San Diego, California, United States 


\title{
Optimised x-ray source scanning trajectories for iterative reconstruction in high cone-angle tomography
}

\author{
Andrew M. Kingston, Glenn R. Myers, Shane J. Latham, Heyang Li, Jan P. Veldkamp, and \\ Adrian P. Sheppard \\ The Australian National University, Research School of Physics and Engineering, \\ Deptartment of Applied Mathematics, Canberra ACT 2601, Australia
}

\begin{abstract}
With the GPU computing becoming main-stream, iterative tomographic reconstruction (IR) is becoming a computationally viable alternative to traditional single-shot analytical methods such as filtered back-projection. IR liberates one from the continuous X-ray source trajectories required for analytical reconstruction. We present a family of novel X-ray source trajectories for large-angle CBCT. These discrete (sparsely sampled) trajectories optimally fill the space of possible source locations by maximising the degree of mutually independent information. They satisfy a discrete equivalent of Tuy's sufficiency condition and allow high cone-angle (high-flux) tomography. The highly isotropic nature of the trajectory has several advantages: (1) The average source distance is approximately constant throughout the reconstruction volume, thus avoiding the differential-magnification artefacts that plague high cone-angle helical computed tomography; (2) Reduced streaking artifacts due to e.g. X-ray beam-hardening; (3) Misalignment and component motion manifests as blur in the tomogram rather than double-edges, which is easier to automatically correct; (4) An approximately shift-invariant point-spread-function which enables filtering as a pre-conditioner to speed IR convergence. We describe these space-filling trajectories and demonstrate their above-mentioned properties compared with a traditional helical trajectories.
\end{abstract}

Keywords: cone beam, computed tomography, micro-tomography, X-ray source trajectory, lattice trajectory, space-filling curve, point spread function, artifact reduction

\section{INTRODUCTION}

$\mathrm{X}$-ray computed tomography $(\mathrm{CT})$ is now a commonplace technique to non-destructively visualise and understand 3D objects. The CTLab at the Australian National University (ctlab.anu.edu.au) performs laboratory based micro-CT employing the fine-focus geometry with micro-focus X-ray sources. Our expertise spans the entire imaging process from micro-CT system hardware design and construction, control and data acquisition software, data processing, tomographic reconstruction, visualization and analysis (e.g. Ref. 1). The major drive of our group has been to achieve quantitative analysis and predictive modeling from the tomograms (e.g. Ref. 2). Thus there is a heavy emphasis on achieving the highest fidelity tomograms in terms of signal-to-noise ratio (SNR) and image sharpness. For cone-beam CT, the ability to achieve this in reasonable scanning time is determined by the source scanning trajectory.

Conventional scanning with a circular X-ray source trajectory around a specimen leads to an approximate reconstruction and is therefore restricted to a low cone-angle geometry; in this case, the detector is only capturing a small solid angle of the available X-ray flux with a consequently low SNR (or long exposure time). It is for this reason that we have been utilising exactly reconstructible helical X-ray source scanning trajectories that enable imaging with large cone-angles (i.e. high-flux). ${ }^{3}$ However, upon implementation, ${ }^{4}$ we found that the helical trajectory has several shortcomings predominantly due to its anisotropic nature: 1) there is nonuniform resolution and noise levels throughout the tomogram; ${ }^{5} 2$ ) the measured data outside the Tam-Danielsson window not utilised in reconstruction is approximately $50 \%$ for $60^{\circ}$ cone-angles; 3) it is highly sensitive to misalignment and results in streaks (rather than blurring as for the circular trajectory) making it difficult to align automatically. ${ }^{6}$ Symmetry can be improved by employing a double-helix trajectory (DHT) ${ }^{5}$ or lowerpitch helices such as the 3PI trajectory. ${ }^{7}$ However, since analytical inversion is utilised, to ensure artifact-free

Corresponding author is A.M.K.: andrew.kingston@anu.edu.au

Developments in X-Ray Tomography X, edited by Stuart R. Stock, Bert Müller, Ge Wang, Proc. of SPIE Vol. 9967, 996712 · ( 2016 SPIE · CCC code: 0277-786X/16/\$18 · doi: 10.1117/12.2238297

Proc. of SPIE Vol. $9967996712-1$ 
tomograms these methods must necessarily acquire significantly more projection images due the the increased scanning path length (including additional overscan).

Traditional X-ray source trajectories are continuous due to either: a) practical reasons such as very rapid (or even continuous) acquisition for the case of medical and synchrotron CT, or b) to enable differentiation as part of analytical reconstruction, such as in filtered back-projections (FBP). Due to the inherently low-flux and relatively long radiograph acquisition times, micro-CT is not restricted by (a) and the employment of iterative reconstruction (IR) schemes removes the need for (b). The development of general purpose GPUs, that are particularly suited to projection/back-projection calculations, has made IR schemes the natural choice even for 3D tomography with large datasets. ${ }^{8}$ CPU reconstruction of large datasets is limited to FBP. To produce high-fidelity tomograms, this then requires a significant amount of preprocessing. Many current generation preprocessing steps are iterative in nature: e.g., geometric alignment by passive autofous, ${ }^{6,9}$ motion correction by reprojection alignment, ${ }^{10,11}$ beam-hardening correction. ${ }^{12-14}$ It is more efficient and effective to incorporate these into an IR scheme. When IR is computationally practical, one is no longer constrained to continuous trajectories.

Our previous work presented at SPIE Developments in X-ray tomography, ${ }^{15}$ explored sparsely sampled lowpitch helical trajectories using a form of IR (specifically: an ordered subset convex algorithm for expectation maximisation of transmission tomography ${ }^{16}$ ). We found that (in terms of sharpness, SNR, residual, and convergence speed) they performed as-well-as or better-than a high-pitch, densely-sampled trajectory for a given scan time. Here, we have taken that concept to the extreme case, where the sample stride length along the helix is comparable to helical pitch. In this limit, the trajectory can no longer be considered continuous, it becomes a lattice tiling of the cylindrical surface upon which the X-ray source can be located, i.e., a space-filling trajectory (SFT).

In Section 2 we present a family of these discrete (or sparsely sampled) trajectories tailored to IR that optimally fill the space of possible source locations. We present the properties of such trajectories in Section 3. In particular, they satisfy a discrete equivalent of Tuy's data sufficiency condition ${ }^{17}$ and allow high cone-angle (high-flux) tomography, maximise the degree of mutually independent information, and the highly isotropic nature of the trajectory provides a more uniform resolution and point-spread-function (PSF). We believe these trajectories can deliver high-fidelity tomograms with the shortest scanning times. We demonstrate the advantages of SFT compared with traditional helical trajectories in Section 4. These include: simple pre-conditioning for rapid IR convergence, reduced artefacts, and enhanced alignment capabilities. We finish with some concluding remarks and an outlook of future work in Section 5. Note that the phantom, system geometry, and noise used for simulations throughout this paper are specified in Appendix A.

\section{THE SPACE-FILLING TRAJECTORY}

The 2D space of possible $\mathrm{X}$-ray source positions is the surface of a cylinder with radius $R$, the source-to-rotationaxis distance, parameterised by $(z, R \theta)$, where $z$ is translation perpendicular to the rotation axis, and $\theta$ is the rotation angle. Instead of considering the scanning trajectory to be a finite sampling of a continuous source trajectory, we define it as a set of distinct points placed in this space. It is natural to consider trajectories for which the points are distributed in a uniform manner throughout the space. We call this type of trajectory a space filling trajectory (SFT). There are numerous practical ways to achieve this type of trajectory, however, care must be taken if one wants to ensure the data sufficiency condition is met; for example, a set of sparse circular trajectories is insufficient. Thus, we have limited our SFT definition here to sparsely sampled (or strided) helices with an extremely low pitch. An example is shown as the black points in Fig. 1 compared with a traditional helical trajectory in red.

\subsection{Reshuffling definition}

An SFT can be generated as a reshuffling of the set of $\theta$-positions from a classical helical trajectory. For example, a trajectory with a pitch of $Z$ and $E$ source points per revolution (subsequently referred to as an ensemble for $\mathrm{SFT})$ has a vector in the source position space of $(Z / E, 2 \pi R / E)$. The angle set can be reordered by striding by $n$ to give the vector $(Z / E, 2 n \pi R / E)$, for $n \in \mathbb{N}$. This results in $n$ revolutions per ensemble or, equivalently, a new pitch of $Z / n$. Restricting $n$ to have a greatest-common-divisor with $E$ of 1 ensures all original angular 


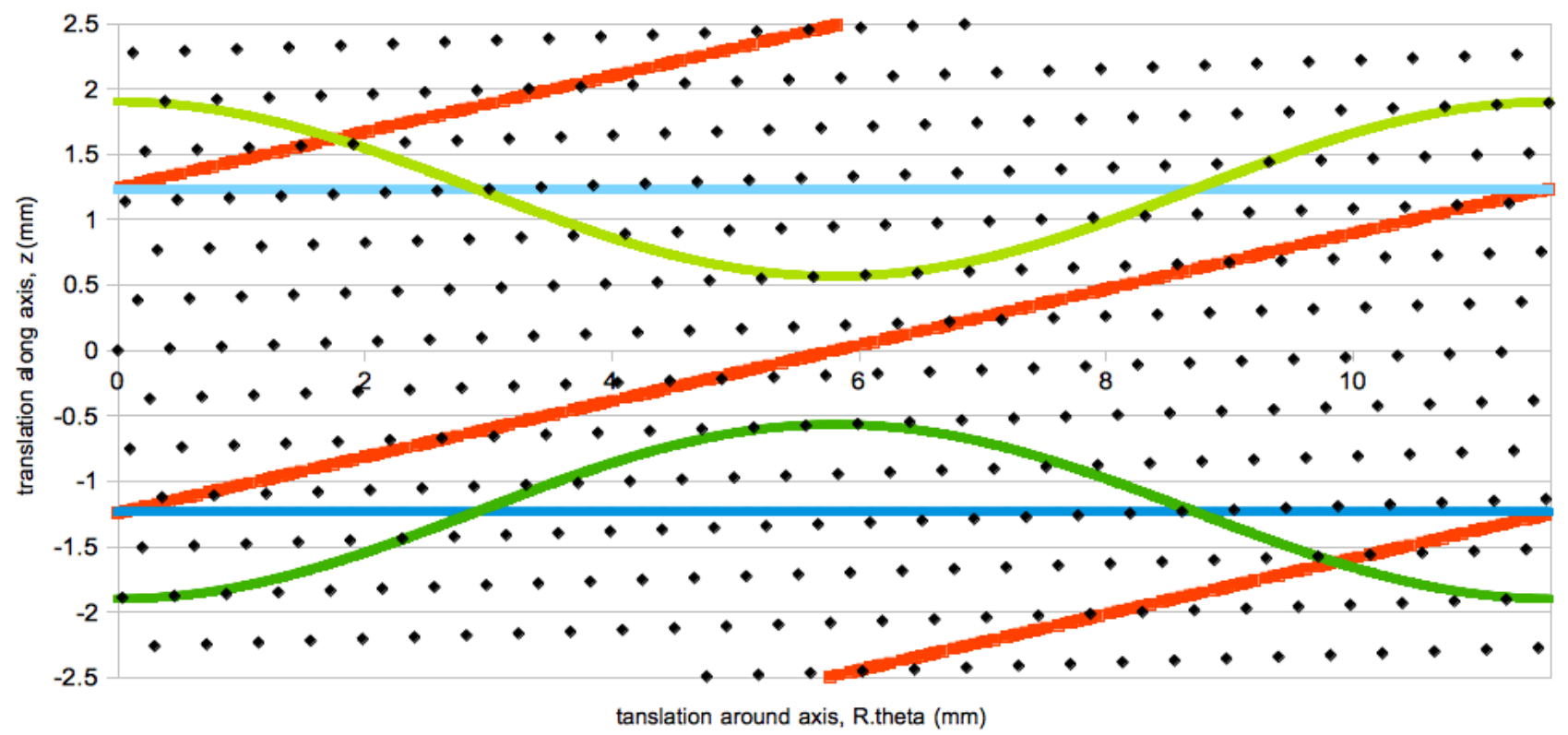

Figure 1. The space of possible X-ray source positions, i.e., the surface of a cylinder with radius $R=1.85 \mathrm{~mm}$; Sourcedetector length is $300 \mathrm{~mm}$, detector width/height is $400 \mathrm{~mm}$ and the number of source points per revolution (or ensemble) is 180. The red points depict the classical helical trajectory. The black points depict the proposed space filling trajectory. The region between the blue lines define the source points seen at the center of the central plane. The region between the green lines define the source points seen at the maximum radius in the central plane.

positions are visited once per ensemble. To obtain approximately equivalent distance between adjacent points along the helix as between successive revolutions, one selects $n$ nearest to:

$$
n=\sqrt{\frac{Z E}{2 \pi R}} .
$$

\subsection{Golden-ratio definition}

Alternatively, we could generate an entirely new set of $\theta$-positions. Let us specify, $Q$ source points per revolution, for $Q \in \mathbb{R}$. The pitch becomes $Q Z / E$. To maximise coverage of the source point plane, one requires source positions to be arranged in a hexagonal tessellation, i.e., a pitch of $\sqrt{3} \pi R / E$ and $Q \sim q+0.5$ for $q \in \mathbb{N}$. If, instead, we set $Q=q+\phi$, where $\phi$ is the golden section $(\sqrt{5}-1) / 2 \approx 0.618$, then we achieve approximate hexagonal tiling and, $\phi$ being irrational ensures that all angles are unique for the entire dataset. Additionally, the partitioning properties of the residual $-\phi 2 \pi R / E$ provide an approximately even sampling of $R \theta$. Therefore, $Q$ is found as:

$$
Q=\left\lfloor\sqrt{\frac{\sqrt{3} \pi E R}{Z}}\right\rfloor+\phi
$$

The vertical translation per ensemble, $Z$, can be defined as the translation that, on average, captures the projection of a given point onto the detector for all radiographs in an ensemble, i.e.,

$$
Z=\frac{H R}{L}
$$

where $H$ is the detector height and $L$ is the source-detector distance. An example space filling trajectory using (3) is depicted by the black points in Fig. 1. 


\section{PROPERTIES OF THE SPACE-FILLING TRAJECTORY}

\subsection{Satisfies data sufficiency}

Not all scanning trajectories provide sufficient information for a theoretically-exact reconstruction. Satisfying the Tuy sufficiency criterion ${ }^{17}$ ensures exact reconstruction is possible from a data sampling perspective. The essence of this criterion is that a trajectory is sufficient if all planes that cut the volume to be reconstructed intersect the scanning trajectory. Of course, this makes sense only when considering continuous scanning trajectories along which the sampling density is approaching the Nyquist criterion, i.e., the angular spacing between adjacent source points is comparable to the angular spacing between adjacent detector pixels. Analytical reconstruction schemes, (e.g., of the FBP type), require such dense sampling along the acquisition path, but iterative reconstruction methods liberate us from this constraint. Here, we propose an equivalent discrete Tuy sufficiency criterion: $a$ trajectory is sufficient if all planes of thickness 1 voxel that intersect the volume to be reconstructed encompass at least one source point. By design, the SFT satisfies this discrete Tuy sufficiency criterion.

\subsection{Greater mutually independent information}

With a large stride between adjacent source points data collected using the SFT contains information about the specimen that is more independent than that for a classical helical trajectory. The approximately hexagonal tiling of the source point plane minimises the area of unpopulated regions and maximises the distance between any two neighbouring source points, making the SFT data maximally independent given the constraints of the imaging apparatus. Tomographic reconstruction from projection data is by nature an ill-posed problem. ${ }^{18}$ The SFT maximally independent projection data should lead to a better conditioned problem which yields reconstructed tomograms with lower levels of noise and geometric artifacts for a given acquisition time. A formal proof that the SFT is less ill-posed than a helical trajectory is an open problem. In what follows we attempt demonstrate by example that the SFT has improved performance with noisy and reduced measured data.

\subsection{Pseudo-uniform resolution}

A helical scanning trajectory is anisotropic, i.e., highly non-uniform when viewed from the perspective of a point within the object. ${ }^{5}$ The number of source points seen by each point (Figs. 2a-i and 2a-ii) and the average distance to the source (Figs. 2b-i and 2b-ii) varies considerably. This leads to non-uniform resolution in the reconstructed volume in high-magnification fine-focus geometry, where geometric magnification across the sample varies significantly (up to a factor of three on the ANU micro-CT systems). Regions of the specimen that are (on average) closest to the source have a much higher magnification than regions that are furthest. By contrast, the number of source points seen by each point for the SFT (Fig. 2a-iii) and the average distance to the source (Fig. 2b-iii) is as uniform as possible.

\subsection{Pseudo-uniform point-spread-function}

The SFT also possesses maximally isotropic point-spread properties, quite distinct from other trajectories where the blurring is directional and location dependent. For the SFT, the directions of the rays passing through each point are as uniformly distributed on the unit sphere as is possible (considering the constraints of the imaging apparatus). An unfiltered back-projection of linearised radiographs, to a good approximation, results in shift-invariant blurring of the volume.

Note that the set of source points seen in back-projection is a function of the position of the voxel within the tomogram. This is due to the limited acceptance-angle specified by the detector-height. A voxel in the center of the tomogram sees source points from all directions equally well, (e.g., the region between the blue lines in Fig. 1), while those seen by a voxel at some non-zero radius is shaped by a sinusoid envelope. For a voxel position $(x, y)$ in the plane, the amplitude of the sinusoid is determined by radius $\left(\sqrt{x^{2}+y^{2}}\right)$, while the phase is determined by $\tan ^{-1}(y / x)$. Fewer near-side points are seen by a voxel than far-side source points, (e.g., the region between the green lines in Fig. 1 for a voxel at maximum radius). The area enclosed by the sinusoidal envelope is unchanged by phase or amplitude which implies that the number of source points seen by all voxels is approximately constant (as can be seen in Fig. 2a-iii). A point cropped at the near side is added at the far side $180^{\circ}$ apart. Here the reshuffling SFT performs slightly worse than the golden ratio SFT definition since the voxel then sees 2 source points that have the same angle but with different $z$ positions. 


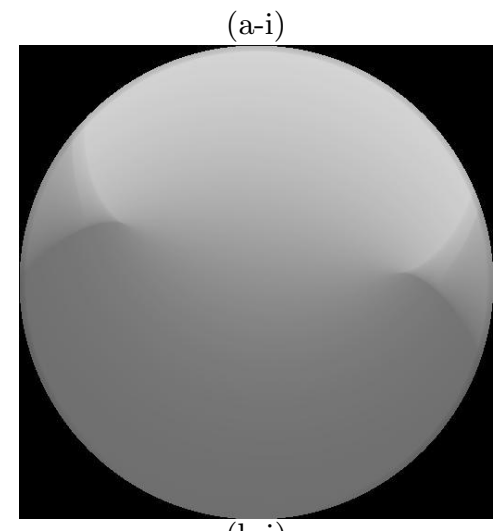

(b-i)
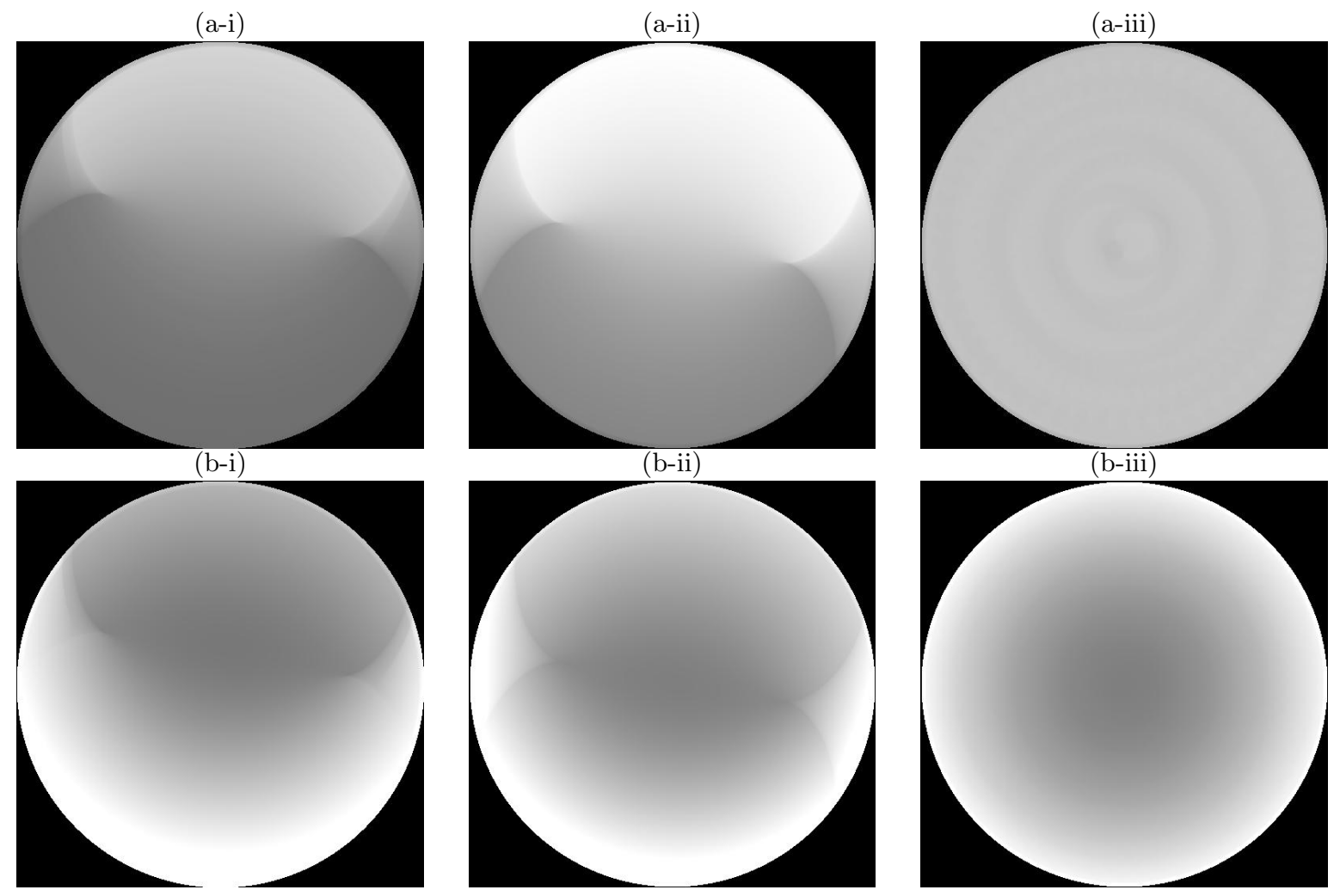

Figure 2. Central 2D horizontal slice through a reconstruction volume showing (a) number of X-ray source positions that back-project through each voxel [black $=0$, white $=941$, and (b) the average X-ray source distance [black $=1.4 \mathrm{~mm}$, white $=2.3 \mathrm{~mm}$, columns are: (i) helical trajectory with the Tam-Danielsson window applied, (ii) unwindowed helical trajectory, and (iii) space filling trajectory. Geometry is specified in Appendix A

\section{ADVANTAGES OF THE SPACE-FILLING TRAJECTORY}

\subsection{Effective pre-conditioning for rapid IR convergence}

In 1980, Colsher ${ }^{19}$ analysed the case of idealised positron emission tomography (PET) data collected with a cylindrical detector and proposed an exact reconstruction algorithm of the back-projection filtration (BPF) and FBP type with shift-invariant spatial filtering. CT data acquired using an SFT is similar to Colsher's idealised PET case, except that SFT contains a discrete set of source positions rather than the quasi-continuum of PET, and it has a planar detector. To utilise the shift-invariant filtering, a Colsher window is applied to the radiographs which enforces a constant acceptance angle:

$$
C(w, h)= \begin{cases}1 & \text { if }|h| \leq(H / 2) \sqrt{\frac{L^{2}+w^{2}}{L^{2}+(W / 2)^{2}}}, \\ 0 & \text { otherwise }\end{cases}
$$

for detector pixel loacted at $(w, h)$ where $L$ is the source-detector-distance, and $W$ and $H$ are the detector width and height respectively. We incorporate modified forms of Colsher filtering in the radiographs or post-backprojection filtering into iterative reconstruction methods to precondition and accelerate convergence.

Figure 3a depicts a direct reconstruction, by 1) Colsher filtering the radiographs, 2) applying the Colsher window to the radiographs, and 3) back-projecting. This filtering technique has been used as a preconditioner in CGM iterative reconstruction in Fig. 3b. The convergence plot in Fig. 3c shows this simple preconditioned CGM (PCGM) gives an order of magnitude speed up in convergence. A volume filtered BPF form that incorporates multi-grid schemes and converges in just 2 iterations is presented in Ref. 20. 
(a)

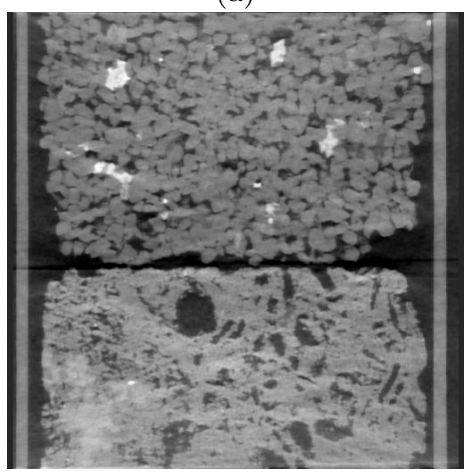

(b)

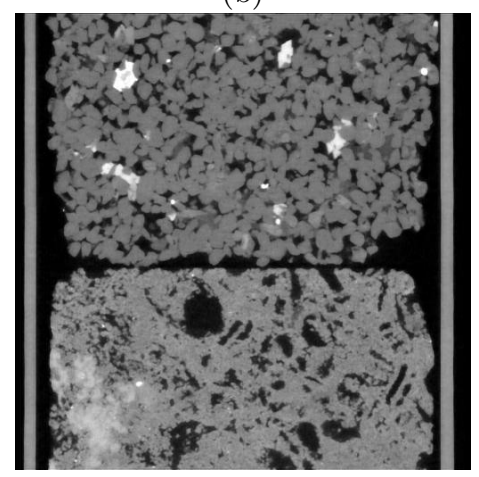

(c)

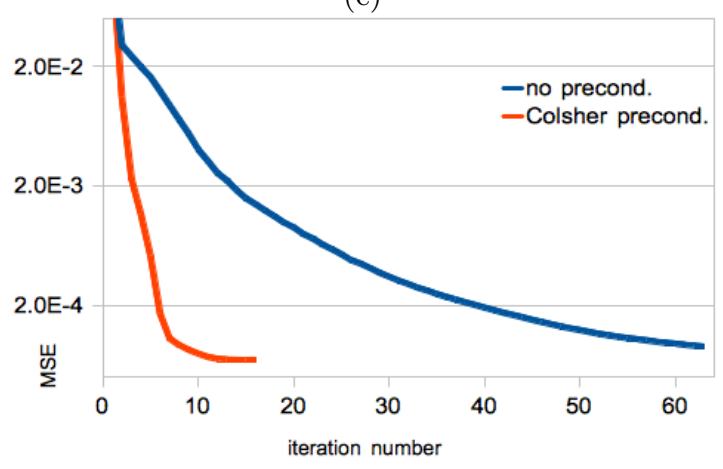

Figure 3. Vertical 2D slices through the $2 \mathrm{~mm}$ diameter sandstone/limestone phantom, reconstructed from a space filling trajectory with a total of 1400 source points, using Colsher-filtered and Colsher-windowed (a) back-projection, (b) PCGM.

\subsection{Improved SNR or reduced scan time and data}

The SFT, which a) satisfies data sufficiency, b) provides maximally independent information, and c) utilises the entire detector area, is a very efficient trajectory. Compared with traditional continuous trajectories, the SFT can provide a tomogram with reduced noise and geometric artifacts from fewer radiographs (or an equivalent quality tomogram in a reduced scan time). Other trajectories, such as DHT and 3PI, that produce informationredundant radiographs (to improve reconstruction quality), still require a high (continuous) sampling rate along the trajectory and thus necessitate more radiographs to produce a high-fidelity tomogram. Figure 4 shows the relative performance of SFT, helical, and circular trajectories given an approximately constant experiment time. It can be seen that the SFT provides the highest fidelity tomogram and has the lowest mean square-error (MSE).

\subsection{Reduced artefacts from beam-hardening and metal inclusions}

The isotropic nature of the SFT has the further advantage that streak artifacts from beam-hardening and metallic inclusions are distributed over the largest possible solid-angle minimising their impact on image quality.

An extreme example is shown in Fig. 5 where a $25.4 \mathrm{~mm}$ diameter pendant made of timber and pewter was imaged with a DHT and SFT. The accelerating voltage of the X-ray source was $120 \mathrm{kV}$ and heavy filtering (1.2 $\mathrm{mm}$ stainless-steel) was applied to the X-rays resulting in a high energy spectrum; the timber is not apparent. The specimen was placed $R=40 \mathrm{~mm}$ from the source, with a $1520 \times 1520$ pixel $(400 \mathrm{~mm} \times 400 \mathrm{~mm})$ detector placed $L=336 \mathrm{~mm}$ from the source and both trajectories used $E=1440$ (ideally $E$ should be 2000). The DHT had a pitch of $Z=51.5 \mathrm{~mm}$ with 4588 source points in total. The SFT used a pitch of $Z=42.5 \mathrm{~mm}$ calculated using (3) [but with $H$ modified considering the Colsher window was applied (4)] with 2989 source points in total. Although artefacts are still visible in the SFT result (and segmentation would still be difficult), there is a significant improvement in image quality.

\subsection{Improved alignment/motion-correction capabilities}

A very important property of the SFT is that it is well behaved when scanner components are misaligned and/or when specimen/component motion occurs during scanning. Kingston et al determined a referenceless post-acquisition software alignment protocol for the circular trajectory in Ref. 9 which optimises tomogram sharpness with respect to the alignment parameters. This protocol is robust for cases where the misalignment manifests as tomogram blurring (as is the case for the circular trajectory and FBP reconstruction). Blur can be easily detected, quantified, and minimised. In contrast, Varslot et al showed that for the helical trajectory with Katsevich filtering, which has no redundancy, misalignment is quite difficult to detect as it introduces geometrical distortions and disjoint features. ${ }^{6}$ This was mitigated by using horizontal ramp-filtering (with no Tam-Danielsson window applied) when scanning for alignment parameter values. By using the full detector data, redundancy was introduced and misalignment manifested largely as blurring. However, some streaking artifacts remained making software alignment using sharpness measures less reliable. 
$(\mathrm{a}-\mathrm{i})$

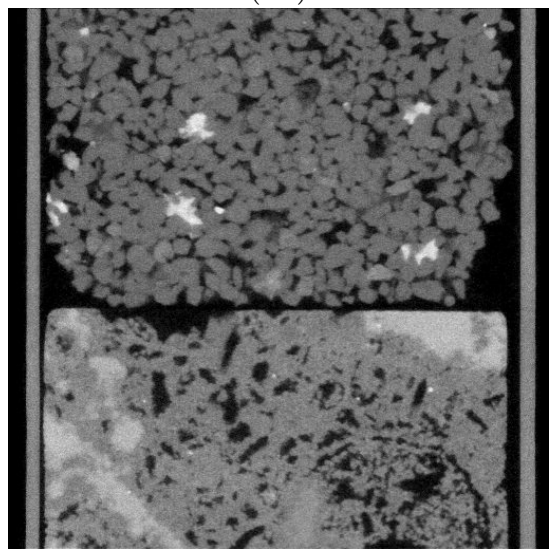

(a-ii)

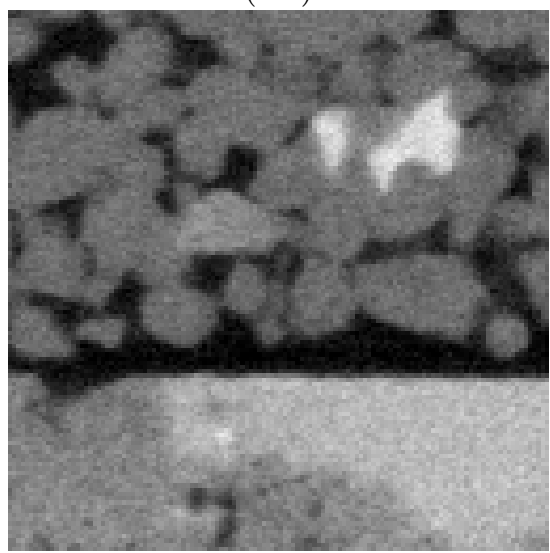

$(\mathrm{b}-\mathrm{i})$

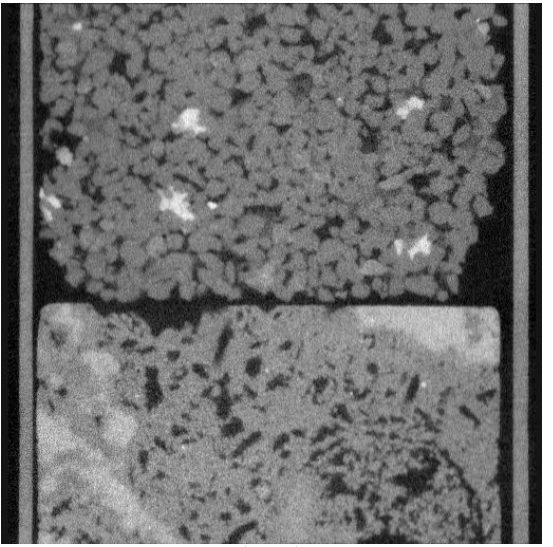

(b-ii)

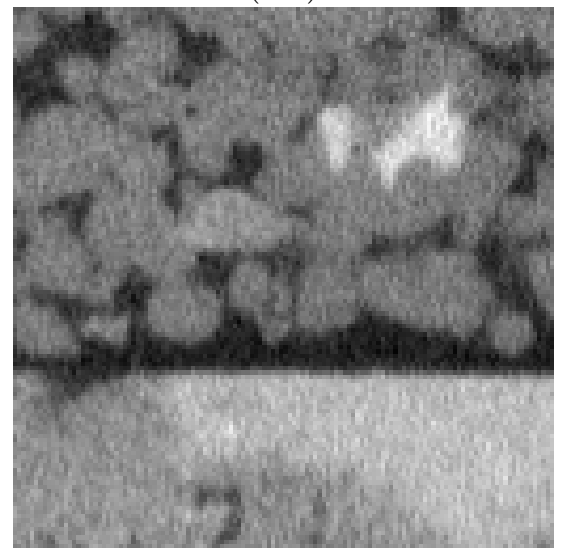

(c-i)

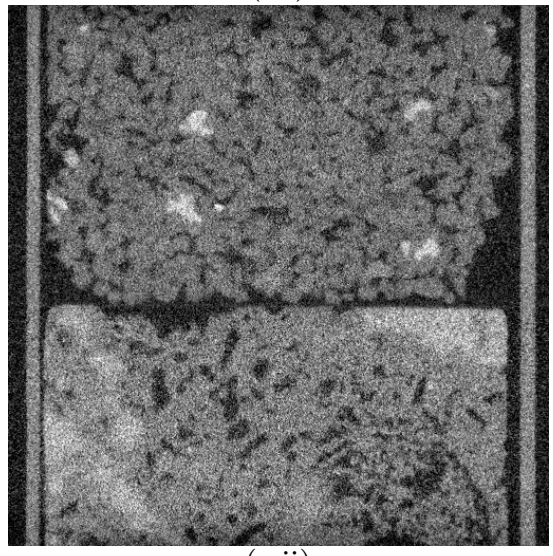

(c-ii)

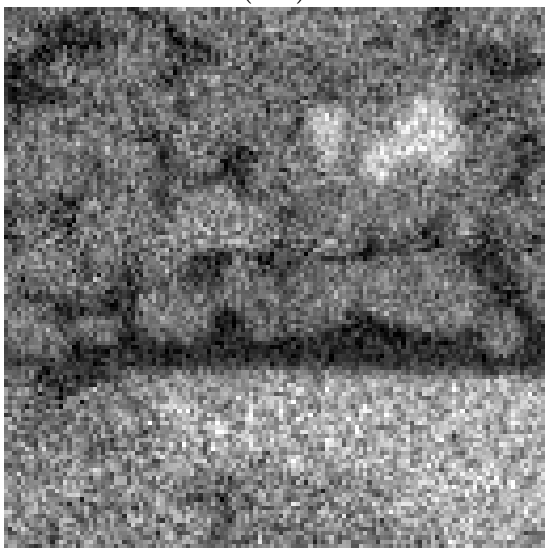

Figure 4. Vertical 2D slices through the $2 \mathrm{~mm}$ diameter sandstone/limestone phantom, reconstructed from data collected over approximately constant experiment time using (a) Space Filling Trajectory with 1400 source points, exposure time $t=16 \mathrm{~s}$. Reconstructed with 512 iterations of SIRT (b) Helical trajectory, with 720 source points per voxel requiring 2800 total source points, $t=8 \mathrm{~s}$. Reconstructed with Katsevich FBP. ${ }^{3}$ (c) Circular trajectory, with $R=7.4 \mathrm{~mm}$ and $L=1200 \mathrm{~mm}$, (i.e. $4 \times$ ), this gives a lower cone-angle of $\pm 9.46^{\circ}$ but has $16 \times$ less flux with $t=32 \mathrm{~s}$ (which is equivalent to $t=2 \mathrm{~s}$ at $L=300 \mathrm{~mm}$ ). Reconstructed with Feldkamp-Davis-Kress FBP. ${ }^{21}$ MSE: (a) 0.0014 (b) 0.0027 (c) 0.0178 . $\left[\right.$ black $=-0.1 \mathrm{~mm}^{-1}$, white $\left.=1.1 \mathrm{~mm}^{-1}\right]$. 
(a)

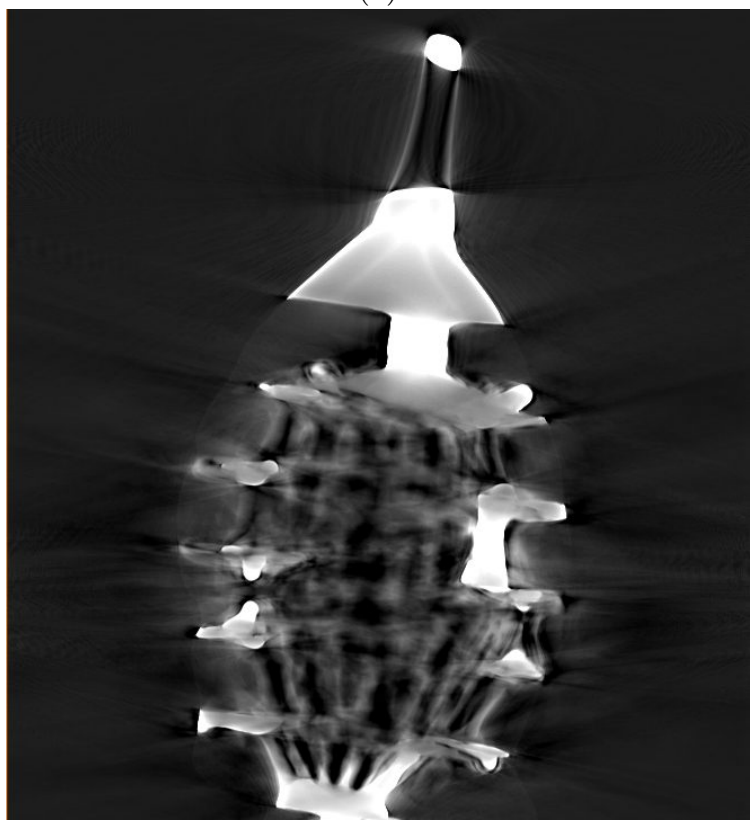

(b)

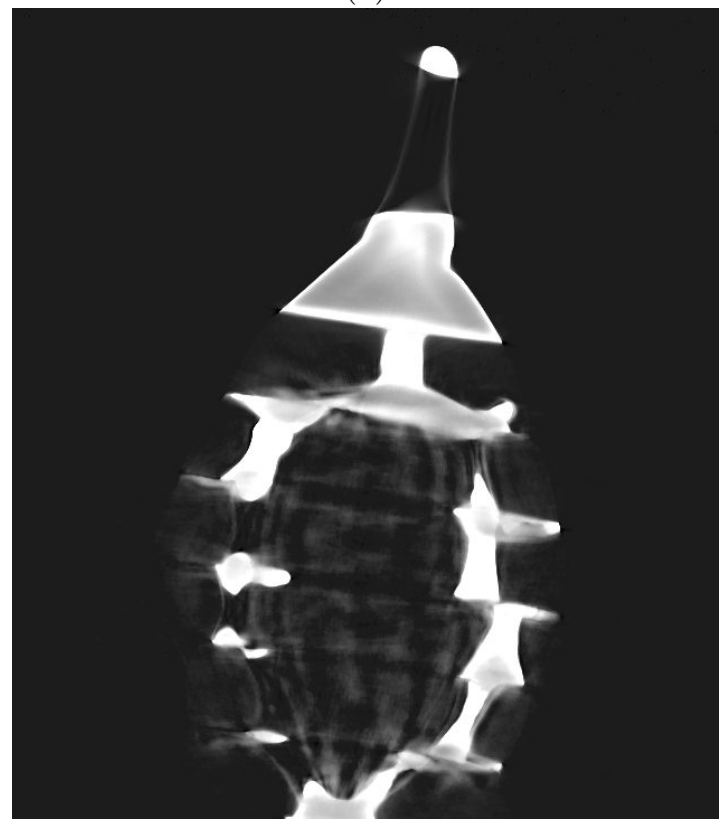

Figure 5. Vertical 2D slices through a $25.4 \mathrm{~mm}$ diameter pendant made of timber injected with pewter. (a) Double-helical trajectory with each helix reconstructed with Katsevich FBP and averaged, (b) Space Filling Trajectory reconstructed using multi-grid BPF method described in Ref. 20. [black $=-0.12 \mathrm{~mm}^{-1}$, white $=0.488 \mathrm{~mm}^{-1}$ ] NB. it is not the same slice presented in each image.

As for the circular trajectory, the isotropic nature of the SFT causes misalignment of radiographs to degrade the tomogram as blur when using horizontal ramp-filtered back-projection. It is an ideal trajectory for software alignment that maximises sharpness in the tomogram (as in Ref. 9). Using the parameterisation and optimal units (ou) defined in Ref. 6, Fig. 6 shows the reconstructions from by ramp-filtered back-projection for a helical trajectory and the SFT produced from radiographs generated with 2ou perturbations of the alignment parameters. The images display the very different nature of the reconstruction artefacts produced by the misalignments for the two trajectories.

The SFT produces similar artefacts when per-radiograph misalignment occurs due to hardware imperfections, such as stage positioning or thermal X-ray source position drift. Since the source rotates so rapidly with time and with $z$-translation, any motion that is smooth in time or with stage translation causes a blurry but geometrically faithful reconstruction. Consequently, reprojected (simulated) radiographs can be unambiguously aligned (registered) with their measure counterparts. Ref. 11 presents an iterative reprojection alignment method where it is shown that their method converges an order of magnitude faster for SFT data than the equivalent helical data.

\section{CONCLUSIONS AND FUTURE WORK}

Iterative reconstruction (IR) schemes do not require a continuous 1D trajectory (such as circular or helical), for which an analytical inversion exists. We have presented a family of discrete (or sparsely sampled) trajectories tailored to IR that optimally fill the space of possible source locations by maximising the degree of mutually independent information. By design the SFT satisfies a discrete equivalent of Tuy's sufficiency condition and allows high cone-angle (high-flux) tomography. We demonstrated that the highly isotropic nature of this trajectory: 1) minimises problems of non-uniform resolution, 2) provides an approximately shift-invariant PSF enabling effective preconditioned IR with fast convergence, 3) minimises artifacts from beam-hardening and metal inclusions, and 4) causes misalignment and motion to be manifest as tomogram blur which can readily be quantified and corrected. We also showed that this trajectory can give higher-fidelity tomograms or provide reduced scan time and data compared with classical helical and circular trajectories. 
(a)

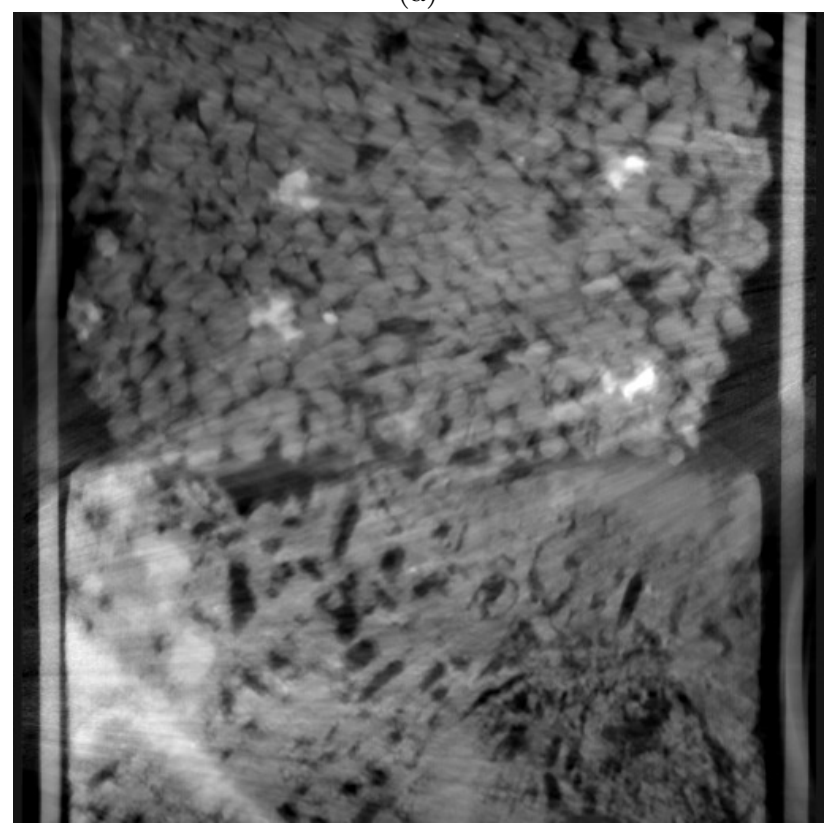

(b)

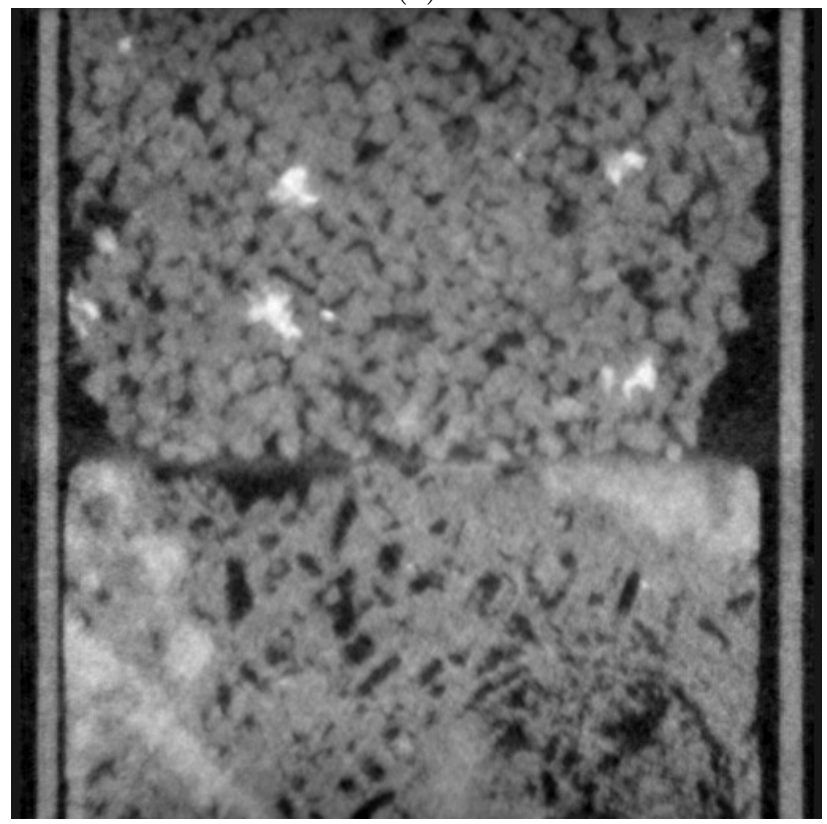

Figure 6. Vertical 2D slices through the $2 \mathrm{~mm}$ diameter sandstone/limestone phantom, with misalignments of $20 u$ in all paramters (as specified in Ref. 6), reconstructed with ramp-filtered back-projection from (a) Helical trajectory, (b) Space Filling Trajectory.

In Ref. 20 we present a rapidly converging multigrid IR algorithm for the SFT and in Ref. 11 we incorporate reprojection alignment motion correction. Our parallel CPU and GPU implementation of projection and backprojection that lays the foundation for this work and makes reconstruction of 100 Giga-voxel datasets practical is

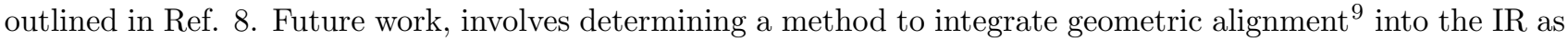
well as beam-hardening correction techniques (such as those in Refs. 12,14) and practical iterative dual-energy techniques (to extend our work in Ref. 22).

\section{APPENDIX A. EXPERIMENTAL (OR SIMULATION) METHOD}

Here we describe the forward-projection simulations, used throughout the paper, performed with various scanning trajectories and noise levels.

\section{A.1 Phantom}

The Phantom volume is generated from a tomogram of stacked sandstone and limestone cores as depicted in Fig. 7. The volume was vertically truncated to a cube and resampled to $N=512$ cubed voxels with a voxel size of $4.11 \mu \mathrm{m}$. Attenuation values ranging from 0.0 to $1.0 \mathrm{~mm}^{-1}$ were posterised into 12 discrete-values.

\section{A.2 Geometry}

Unless otherwise specified, the geometry used for simulation is as follows: the phantom is placed at $R=1.85 \mathrm{~mm}$ with a $400 \times 400 \mathrm{~mm}^{2}$ detector placed at $L=300 \mathrm{~mm}$. To avoid the inverse crime and simulate the physical X-ray paths more realistically, the forward-projection data was calculated with an $1800 \times 1800$ pixel detector that was rebinned to $600 \times 600$ pixels. We have assumed $E=720$.

For helical trajectories, $Z=2.485 \mathrm{~mm}$ has been used to maximise the Tam-Danielsson window on the detector (see Fig. 8a), and a conservative overscan of $\pi / 4+3 \alpha_{f}$ where $\alpha_{f}$ is the fan-angle. For SFT, $Z=2.47 \mathrm{~mm}$ calculated according to (3), with an overscan of $E / 2$ radiographs. The Colsher window for this trajectory is shown in Fig. 8b. 
(a)

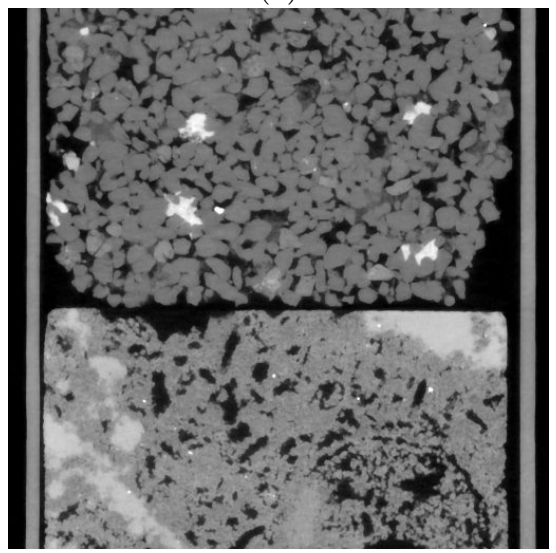

(b)

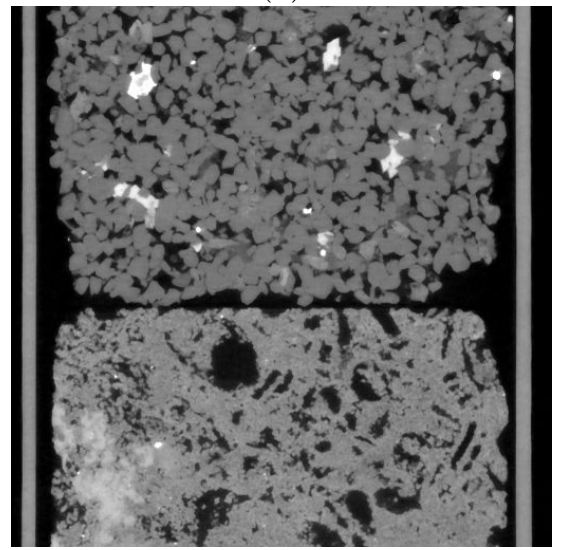

(c)

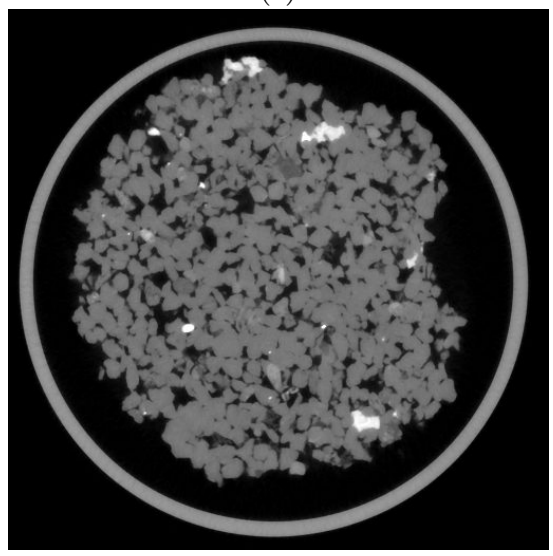

Figure 7. Central 2D slices through the 3D $2 \mathrm{~mm}$ diameter sandstone/limestone phantom used for simulations throughout this paper. (a) $x$-slice, (b) $y$-slice, (c) $z$-slice. $\left[\right.$ black $=0.0 \mathrm{~mm}^{-1}$, white $=1.0 \mathrm{~mm}^{-1}$ ]

\section{A.3 Noise}

When noise is included, it is parameterised by exposure time, $t$ in seconds. A mean $\mathrm{X}$-ray energy of $\xi=30 \mathrm{keV}$ is assumed, and a typical detector conversion efficiency of $\tau=0.016$ is used. We define measured intensity to be $\gamma=1024$ intensity units (IU) per second, according to $\gamma=n \xi \tau$, where $n$ is the number of photons. Assuming a Poisson distribution, the variance of measurement is $\sigma^{2}=n \xi^{2} \tau^{2}$. We have also included dark current noise assuming a normal distribution with a typical value $\sigma_{d}^{2}=24 \mathrm{IU}$.

(a)
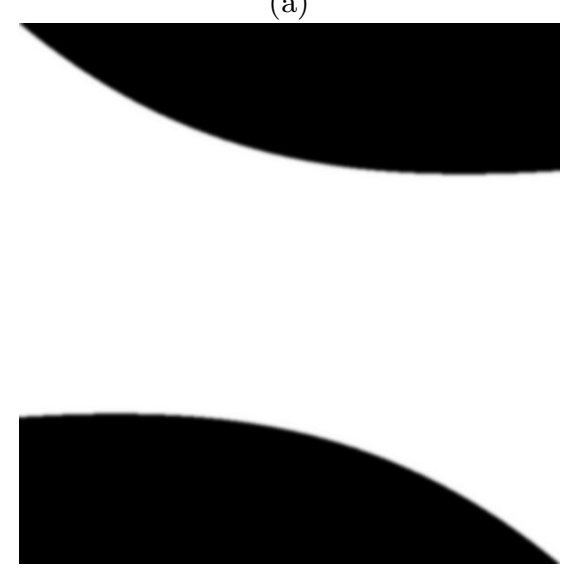

(b)

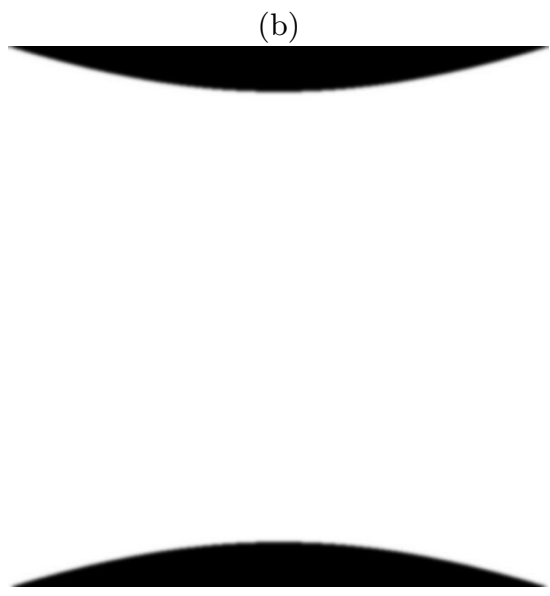

Figure 8. The windows that may be appled to projections according to the geometry specified in Sect. A. (a) The Tam-Danielsson for the helical trajectory, (b) The Colsher window for the space-filling trajectory.

\section{ACKNOWLEDGMENTS}

The authors acknowledge funding and support through the companies involved in the Digicore consortium as well as the Australian Research Council (ARC) linkage grant LP150101040 with FEI Company. APS acknowledges support from the ARC through Future Fellowship FT100100470.

\section{REFERENCES}

[1] Sheppard, A., Latham, S., Middleton, J., Kingston, A., Myers, G., Varslot, T., Fogden, A., Sawkins, T., Cruikshank, R., Saadatfar, M., Francois, N., Arns, C., and Senden, T., "Techniques in helical scanning and 
dynamic imaging and image segmentation for improved quantitative analysis with x-ray micro-ct," Nuclear Instruments and Methods in Physics Research: Section B 324, 49-56 (2014).

[2] Francois, N., Saadatfar, M., Cruikshank, R., and Sheppard, A., "Geometrical frustration in amorphous and partially crystallized packings of spheres," Physical Review Letters 111(14), 148001 (2013).

[3] Katsevich, A., "Theoretically exact filtered backprojection-type inversion algorithm for spiral ct," SIAM Journal of Applied Math. 62(6), 2012-2026 (2002).

[4] Varslot, T., Kingston, A., Sheppard, A., and Sakellariou, A., "Fast high-resolution micro-ct with exact reconstruction methods," Proc. SPIE Developments in X-ray Tomography VII 7804, 7804.13 (2010).

[5] Varslot, T., Kingston, A., Myers, G., and Sheppard, A., "Considerations for high-magnification high-coneangle helical micro-ct," Proc. SPIE Developments in X-ray Tomography VIII 8506, 8506.14 (2012).

[6] Varslot, T., Kingston, A., Myers, G., and Sheppard, A., "High-resolution helical cone-beam micro-ct with theoretically-exact reconstruction from experimental data," Medical Physics 38(10), 5459-5476 (2011).

[7] Katsevich, A., "3pi algorithms for helical computer tomography," Advances in Applied Mathematics 36(3), 213-250 (2006).

[8] Myers, G., Latham, S., Kingston, A., Varslot, T., and Sheppard, A., "High cone-angle x-ray computed micro-tomography with 90 gigavoxel datasets," Proc. SPIE Developments in X-ray Tomography X 9967 (2016).

[9] Kingston, A., Sakellariou, A., Varslot, T., Myers, G., and Sheppard, A., "Reliable automatic alignment of tomographic projection data by passive auto-focus," Medical Physics 38(9), 4934-4945 (2011).

[10] Mayo, S., Miller, P., Gao, D., and Sheffield-Parker, J., "Software image alignment for x-ray microtomography with submicrometre resolution using a sem-based x-ray microscope," Journal of microscopy 228(3), 257-263 (2007).

[11] Latham, S., Kingston, A., Recur, B., Myers, G., and Sheppard, A., "Multi-resolution radiograph alignment for motion correction in x-ray micro-tomography," Proc. SPIE Developments in X-ray Tomography X $\mathbf{9 9 6 7}$ (2016).

[12] Van Gompel, G., Van Slambrouck, K., Defrise, M., Batenburg, K. J., de Mey, J., Sijbers, J., and Nuyts, J., "Iterative correction of beam hardening artifacts in ct," Medical Physics 38(S1), S36-S49 (2011).

[13] Kingston, A. M., Myers, G. R., and Varslot, T. K., "X-ray beam hardening correction by minimizing reprojection distance," Proc. SPIE Developments in X-ray Tomography VIII 8506, 85061D:1-10 (2012).

[14] De Man, B., Nuyts, J., Dupont, P., Marchal, G., and Suetens, P., "An iterative maximum-likelihood polychromatic algorithm for ct," IEEE Transactions on Medical Imaging 20(10), 999-1008 (2001).

[15] Recur, B., Fauconneau, M., Kingston, A., Myers, G., and Sheppard, A., "Iterative reconstruction optimisations for high angle cone-beam micro-ct," Proc. SPIE Developments in X-ray Tomography IX $\mathbf{9 2 1 2}$, 921214:1-12 (2014).

[16] Erdogan, H. and Fessler, J. A., "Ordered subsets algorithms for transmission tomography," Physics in medicine and biology 44(11), 2835 (1999).

[17] Tuy, H., "An inverse formula for cone-beam reconstruction," SIAM Journal of Applied Math. 43, 546-552 (1983).

[18] Natterer, F., [The mathematics of computerized tomography], vol. 32, Siam (1986).

[19] Colsher, J., "Fully-three-dimensional positron emission tomography," Physics in Medicine and Biology 25(1), 103-115 (1980).

[20] Myers, G., Kingston, A., Latham, S., Recur, B., Turner, M., Beeching, L., and Sheppard, A., "Rapidlyconverging multigrid reconstruction of cone-beam tomographic data," Proc. SPIE Developments in X-ray Tomography X 9967 (2016).

[21] Feldkamp, L., Davis, L., and Kress, J., "Practical cone-beam algorithm," Journal of the Optical Society of America A 1(6), 612-619 (1984).

[22] Recur, B., Paziresh, M., Myers, G., Kingston, A., Latham, S., and Sheppard, A., "Dual-energy iterative reconstruction for material characterisation," Proc. SPIE Developments in X-ray Tomography IX 9212, 921213:1-9 (2014). 